PROCEEDINGS OF THE

AMERICAN MATHEMATICAL SOCIETY

Volume 140, Number 10, October 2012, Pages 3453-3462

S 0002-9939(2012)11233-X

Article electronically published on February 16, 2012

\title{
ON THE ERGODICITY OF CONFORMAL MEASURES FOR RATIONAL MAPS WITH TOTALLY DISCONNECTED JULIA SETS
}

\author{
YU ZHAI \\ (Communicated by Bryna Kra)
}

\begin{abstract}
Let $f$ be a non-hyperbolic rational map with totally disconnected Julia set whose Fatou set is an attracting domain. In this paper, we prove that the number of ergodic components of any conformal measure for $f$ is bounded by the number of critical points in its Julia set.
\end{abstract}

\section{InTRODUCTION AND STATEMENT}

Let $f$ be a rational map from the Riemann sphere $\widehat{\mathbb{C}}$ onto itself of degree $d \geqslant 2$. The Fatou set $F(f)$ and the Julia set $J(f)$ of $f$ represent the stable part and the chaotic part of the complex dynamical system respectively. For the background of a complex dynamical system, readers can refer to the book [8. Measurable dynamics is an important field in complex dynamics; it studies measures and dimensions relative to the Julia sets, among which conformal measure is a very remarkable one.

Definition 1.1. A probability measure $\mu$ supported on $J(f)$ is called a $\delta$-conformal measure $(0<\delta \leqslant 2)$ for $f$ if the equation

$$
\mu(f(A))=\int_{A}\left|f^{\prime}(z)\right|^{\delta} d \mu
$$

holds for every Borel set $A \subset J(f)$ such that $\left.f\right|_{A}$ is injective.

The existence of conformal measure for any rational map was shown in [16] by Sullivan; see also [4. Clearly, if $J(f)$ has positive two-dimensional Lebesgue measure, then the Lebesgue measure on $J(f)$ is a 2-conformal measure for $f$. According to the construction of the conformal measure, all the conformal measures form a closed set in the sense of weak convergence, so there exists a conformal measure whose exponent is the infimum of all the exponents of conformal measures.

Definition 1.2. A conformal measure $\mu$ for $f$ is said to be ergodic if $\mu(X)=0$ or $\mu(X)=1$ whenever $X \subset J(f)$ is $f$-invariant, namely, $X=f^{-1}(X)$.

Received by the editors October 22, 2010 and, in revised form, April 6, 2011.

2010 Mathematics Subject Classification. Primary 37F10, 37F20; Secondary 28D99.

Key words and phrases. Conformal measure, ergodic component, Branner-Hubbard puzzle, KSS nest.

The author was supported in part by China Postdoctoral Science Foundation Grant \#20080440543.

(C)2012 American Mathematical Society Reverts to public domain 28 years from publication 3453 
Many efforts were made for proving the ergodicity of the conformal measures. For any hyperbolic rational map $f$, in [16], Sullivan proved that there is only one conformal measure $\mu$ supported on $J(f)$, whose exponent $\delta$ equals the Hausdorff dimension of $J(f)$. This conformal measure is ergodic; moreover, the $\delta$-dimensional Hausdorff measure of $J(f)$ is finite and non-zero, equivalent to the $\delta$-conformal measure $\mu$. In fact, by the uniformly hyperbolicity of $f$ in $J(f)$, one can deduce that the $\mu$-measure of the small ball $B$ centered on $J(f)$ is comparable to $(\operatorname{diam} B)^{\delta}$. The analytical tool is the distortion theorem for univalent maps.

There is a class of rational maps with weaker hyperbolicity called parabolic rational maps, whose Julia sets contain parabolic periodic points but no critical points. For a parabolic rational map $f$, the action of $f$ in $J(f)$ is expansive but not expanding. The ergodicity of conformal measures for such maps is proved by Denker and Urbański; see [1] and [5].

If the Julia set of a rational map contains critical points, the analysis becomes more difficult. The critical points destroy the structure of the Julia sets. We will encounter the trouble that numerous orbits accumulate to the critical points, and we have to find suitable holomorphic inverse branches and control the measure.

In the unicritical case, Prado proved that conformal measures for several classes of unimodal polynomials are ergodic, such as finitely renormalizable quadratic polynomials, infinitely renormalizable quadratic polynomials with a priori bounds, unimodal polynomials with real coefficients and so on; see [12.

In the multicritical case, however, it is usually difficult to obtain the ergodicity of arbitrary conformal measures. But for some special ones, the ergodicity still holds.

The conformal measures with the minimum exponent are the most important ones among all the conformal measures. The ergodicity of such a kind of conformal measure for summable rational maps and topological Collet-Eckmann rational maps was solved in [6] and [13], respectively. Urbański proved the ergodicity of the conformal measures whose exponent equals the Hausdorff dimension of the Julia set for rational maps with only non-recurrent critical points in their Julia sets; see 17. To learn some other results in special cases, readers can refer to [3, 14] or the survey paper 18 .

In the general case, we can only achieve a weaker ergodicity sometimes; that is, the conformal measure has only finitely many ergodic components; see [1] for example.

There is also some progress on this ergodic problem for meromorphic functions and entire functions; see [9], 10] and [19.

In this paper, we focus on the rational map with totally disconnected Julia set, whose Fatou set has only one component. The Fatou set of such a rational map is either an attracting domain or a parabolic domain. In other words, there is either an attracting fixed point in $F(f)$ or a parabolic fixed point in $J(f)$. Here, we study the ergodicity of conformal measures for the rational maps in the attracting case by combinatorial and analytical methods.

Let $\mathcal{F}$ be the set of non-hyperbolic rational maps $f$ satisfying the following properties:

(1) $\operatorname{deg} f \geqslant 2$;

(2) $J(f)$ is totally disconnected;

(3) $F(f)$ is an attracting domain. In other words, there is an attracting fixed point in $F(f)$. 
We prove the following.

Main Theorem. Let $f \in \mathcal{F}$. Then the number of ergodic components of any conformal measure for $f$ is bounded by the number of critical points in $J(f)$.

Immediately, we have the following.

Corollary. Let $f \in \mathcal{F}$. If there is only one critical point in $J(f)$, then any conformal measure of $f$ is ergodic.

\section{BRANNER-HUBBARD PUZZLE AND KSS NEST}

The combinatorial tools we used in this paper are the Branner-Hubbard puzzle and the KSS nest invented by Kozlovski, Shen and van Strien for solving the quasiconformal rigidity of real polynomials.

First, we construct the Branner-Hubbard puzzle for $f \in \mathcal{F}$. We assume that $\infty$ is the attracting fixed point for $f$.

Take a simply connected neighborhood $U_{0} \subset F(f)$ of $\infty$ such that $\overline{U_{0}} \subset f^{-1}\left(U_{0}\right)$. In particular, we can take the disk $\{z:|z|>R\}$ as $U_{0}$, where $R$ is a sufficiently large number and $\{z:|z|=R\}$ is disjoint from the critical orbits of $f$. Let $U_{n}$ be the component of $f^{-n}\left(U_{0}\right)$ containing $\infty$. Then $\overline{U_{n}} \subset U_{n+1}$ and $F(f)=\bigcup_{n=0}^{\infty} U_{n}$. For an integer $N_{0}$ large enough, $f^{-n}\left(U_{N_{0}}\right)$ has only one component for any $n \geqslant 0$. The set $f^{-n}\left(\widehat{\mathbb{C}}-\overline{U_{N_{0}}}\right)$ is a disjoint union of finitely many topological disks. For each $n \geqslant 0$, let $\mathbf{P}_{\mathbf{n}}$ be the collection of all the components of $f^{-n}\left(\widehat{\mathbb{C}}-\overline{U_{N_{0}}}\right)$, which are called puzzle pieces of depth $n$. For two arbitrary different puzzle pieces $P_{1}$ and $P_{2}$, there are three possibilities: $\overline{P_{1}} \cap \overline{P_{2}}=\emptyset, \overline{P_{1}} \subset P_{2}$ or $P_{1} \supset \overline{P_{2}}$.

For any point $x \in J(f)$ and any $n \geqslant 0$, there is only one puzzle piece $P_{n}(x) \in \mathbf{P}_{\mathbf{n}}$ containing $x$. Thus each point $x \in J(f)$ determines a nested sequence $P_{0}(x) \supset$ $P_{1}(x) \supset \cdots$ and $\bigcap_{n \geqslant 0} P_{n}(x)=\{x\}$, since $J(f)$ is totally disconnected.

Take $N_{0}$ large enough such that $U_{N_{0}}$ contains all the critical points in $F(f)$ and each puzzle piece contains at most one critical point.

We say that a critical point is recurrent if $c \in \omega(c)$, where $\omega(c)$ is the set of limit points of the forward orbit of $c$. Otherwise, $c$ is called non-recurrent.

Let

$$
\begin{gathered}
\text { Crit }=\{c \in J(f): c \text { is a critical point of } f\}, \\
\text { Crit }_{0}=\{c \in J(f): c \text { is a recurrent critical point of } f\} .
\end{gathered}
$$

Definition 2.1. (1) We say that $x$ is combinatorially convergent to $y$, written as $x \rightarrow y$, if for any $n \geqslant 0$ there exists $j>0$ such that $f^{j}(x) \in P_{n}(y)$. It is clear that $x \rightarrow y$ if and only if $y \in \bigcup_{n \geqslant 1} f^{-n}(x)$ or $y \in \omega(x)$, the limit set of the forward orbit of $x$. If $x \rightarrow y$ and $y \rightarrow z$, then $x \rightarrow z$. For each critical point $c \in$ Crit, let

$$
[c]=\left\{c^{\prime} \in \text { Crit }: c \rightarrow c^{\prime} \text { and } c^{\prime} \rightarrow c\right\} .
$$

$[c]$ is called the combinatorially equivalent class of $c$.

(2) We say that $x$ is non-critical if $x \rightarrow c$ for any $c \in$ Crit.

(3) We say that $P_{n+k}\left(c^{\prime}\right)$ is a child of $P_{n}(c)$ if $c^{\prime} \in[c], f^{k}\left(P_{n+k}\left(c^{\prime}\right)\right)=P_{n}(c)$, and $f^{k-1}: P_{n+k-1}\left(f\left(c^{\prime}\right)\right) \rightarrow P_{n}(c)$ is conformal.

(4) Suppose that $c \rightarrow c$, i.e. $[c] \neq \emptyset$. We say that $c$ is persistently recurrent if $P_{n}\left(c_{1}\right)$ has only finitely many children for all $n \geqslant 0$ and all $c_{1} \in[c]$. Otherwise, $c$ is said to be reluctantly recurrent. 
Remark 2.2. We say that a critical point $c$ is combinatorially recurrent if $c \rightarrow c$. This terminology is equivalent to recurrent in the usual sense that $c \in \omega(c)$ when $J(f)$ is totally disconnected.

Remark 2.3. We consider the preperiodic critical points as non-recurrent ones since their orbits are finite. All the statements and proofs about non-recurrent critical points are available for preperiodic critical points.

While we construct the Branner-Hubbard puzzle piece, we take $N_{0}$ large enough if necessary to make sure that for each $c \in$ Crit, $f^{n}(c) \notin P_{0}\left(c^{\prime}\right)$ holds for any $n \geqslant 0$ and any $c^{\prime} \in$ Crit if $c \nrightarrow c^{\prime}$.

Let

$$
\begin{aligned}
& \text { Crit }_{\mathrm{n}}=\{c \in \text { Crit }: c \text { is non-critical }\}, \\
& \text { Crit }_{\mathrm{p}}=\{c \in \text { Crit }: c \text { is persistently recurrent }\}, \\
& \text { Crit }_{\mathrm{r}}=\{c \in \text { Crit }: c \text { is reluctantly recurrent }\}, \\
& \text { Crit }_{\mathrm{en}}=\left\{c^{\prime} \in \text { Crit }: c^{\prime} \nrightarrow c^{\prime} \text { and } c^{\prime} \rightarrow c \text { for some } c \in \mathrm{Crit}_{\mathrm{n}}\right\}, \\
& \text { Crit }_{\mathrm{ep}}=\left\{c^{\prime} \in \text { Crit }: c^{\prime} \nrightarrow c^{\prime} \text { and } c^{\prime} \rightarrow c \text { for some } c \in \mathrm{Crit}_{\mathrm{p}}\right\}, \\
& \text { Crit }_{\mathrm{er}}=\left\{c^{\prime} \in \text { Crit }: c^{\prime} \nrightarrow c^{\prime} \text { and } c^{\prime} \rightarrow c \text { for some } c \in \mathrm{Crit}_{\mathrm{r}}\right\} .
\end{aligned}
$$

Then

$$
\text { Crit }=\text { Crit }_{n} \cup \text { Crit }_{p} \cup \text { Crit }_{\mathrm{r}} \cup \text { Crit }_{\text {en }} \cup \text { Crit }_{\text {ep }} \cup \text { Crit }_{\text {er }} .
$$

This is not a classification because these sets might intersect.

Let $A$ be an open set and $x \in A$. The connected component of $A$ containing $x$ will be denoted by $\operatorname{Comp}_{x} A$. Given a puzzle piece $I$, let

$$
D(I)=\left\{z \in \mathbb{C}: \text { There exists } k \geqslant 1 \text { such that } f^{k}(z) \in I\right\}=\bigcup_{k \geqslant 1} f^{-k}(I) .
$$

For any $z \in D(I)$, let $k \geqslant 1$ be the smallest integer such that $f^{k}(z) \in I$ and let $n_{0}$ be the depth of $I$. Then there is at most one piece in

$$
\left\{P_{n_{0}+k}(z)=\operatorname{Comp}_{z} f^{-k}(I), f\left(P_{n_{0}+k}(z)\right), \ldots, f^{k-1}\left(P_{n_{0}+k}(z)\right)\right\}
$$

which contains a critical point $c$ for any $c \in$ Crit. Hence

$$
\operatorname{deg}\left(f^{k}: P_{n_{0}+k}(z) \rightarrow I\right) \leqslant D
$$

for a constant $D<\infty$ depending only on Crit.

A puzzle piece is called critical if it contains a critical point. In [7, Kozlovski, Shen and van Strien have constructed a nest of critical puzzle pieces around persistently recurrent critical points. Such a nest will be called a KSS nest. The detailed process of constructing a KSS nest is omitted in our paper, but can be found in [7] and [15].

Suppose $c_{0}$ is a persistently recurrent critical point. Let

$$
b=\#\left[c_{0}\right], d_{0}=\operatorname{deg}_{c_{0}} f, d_{\max }=\max \left\{\operatorname{deg}_{c} f: c \in\left[c_{0}\right]\right\},
$$

and

$$
\operatorname{orb}\left(\left[c_{0}\right]\right)=\bigcup_{n \geqslant 0} f^{n}\left(\left[c_{0}\right]\right) .
$$

Roughly speaking, the KSS nest is a nested sequence of critical puzzle pieces $K_{0}\left(c_{0}\right) \supset K_{1}\left(c_{0}\right) \supset \cdots \supset K_{n}\left(c_{0}\right) \supset \cdots$ around $c_{0}$ such that

$$
\operatorname{deg}\left(f^{p_{n}}: K_{n}\left(c_{0}\right) \rightarrow K_{n-1}\left(c_{0}\right)\right) \leqslant D_{0},
$$


where $D_{0}<\infty$ is a constant depending only on $b$ and $d_{\max }$. Note that the subscript $n$ does not stand for the depth of the puzzle piece in the KSS nest.

In [7, the authors also found a puzzle piece $\widetilde{K}_{n}\left(c_{0}\right) \supset K_{n}\left(c_{0}\right)$ for each $n$ such that

$$
\left(\widetilde{K}_{n}\left(c_{0}\right)-K_{n}\left(c_{0}\right)\right) \cap \operatorname{orb}\left(\left[c_{0}\right]\right)=\emptyset .
$$

Furthermore, by means of the combinatorial information of the KSS nest indicated in [7] and [15], Qiu and Yin proved the following key lemma about the estimate of the modulus for the KSS nest.

Lemma 2.4 ([15]). For any $c_{0} \in \mathrm{Crit}_{\mathrm{p}}$, there exists a constant $m>0$ depending only on $b$ and $d_{\text {max }}$, such that $\left.\bmod \left(\widetilde{K}_{n}\left(c_{0}\right)-\overline{K_{n}\left(c_{0}\right.}\right)\right) \geqslant m$ for all $n \geqslant 1$.

\section{Density estimates associated to PuZzle pieces}

For any $x \in J(f)$, let

$$
\begin{aligned}
& \operatorname{Crit}(x)=\{c \in \operatorname{Crit}: x \rightarrow c\}, \\
& \operatorname{Crit}_{\mathrm{n}}(x)=\{c \in \operatorname{Crit}(x): c \text { is non-critical }\}, \\
& \operatorname{Crit}_{\mathrm{p}}(x)=\{c \in \operatorname{Crit}(x): c \text { is persistently recurrent }\}, \\
& \operatorname{Crit}_{\mathrm{r}}(x)=\{c \in \operatorname{Crit}(x): c \text { is reluctantly recurrent }\}, \\
& \operatorname{Crit}_{\mathrm{en}}(x)=\left\{c^{\prime} \in \operatorname{Crit}(x): c^{\prime} \nrightarrow c^{\prime} \text { and } c^{\prime} \rightarrow c \text { for some } c \in \operatorname{Crit}_{\mathrm{n}}(x)\right\}, \\
& \operatorname{Crit}_{\mathrm{ep}}(x)=\left\{c^{\prime} \in \operatorname{Crit}(x): c^{\prime} \nrightarrow c^{\prime} \text { and } c^{\prime} \rightarrow c \text { for some } c \in \operatorname{Crit}_{\mathrm{p}}(x)\right\}, \\
& \operatorname{Crit}_{\mathrm{er}}(x)=\left\{c^{\prime} \in \operatorname{Crit}(x): c^{\prime} \nrightarrow c^{\prime} \text { and } c^{\prime} \rightarrow c \text { for some } c \in \operatorname{Crit}_{\mathrm{r}}(x)\right\} .
\end{aligned}
$$

Then

$$
\operatorname{Crit}(x)=\operatorname{Crit}_{\mathrm{n}}(x) \cup \operatorname{Crit}_{\mathrm{p}}(x) \cup \operatorname{Crit}_{\mathrm{r}}(x) \cup \operatorname{Crit}_{\mathrm{en}}(x) \cup \operatorname{Crit}_{\mathrm{ep}}(x) \cup \operatorname{Crit}_{\mathrm{er}}(x) .
$$

Further let

$$
\begin{aligned}
& J_{1}=\left\{x \in J(f): \operatorname{Crit}(x)=\emptyset \operatorname{or}_{C} \operatorname{Crit}_{\mathrm{n}}(x) \cup \operatorname{Crit}_{\mathrm{p}}(x) \neq \emptyset\right\}, \\
& J_{2}=\left\{x \in J(f): \operatorname{Crit}(x)=\operatorname{Crit}_{\mathrm{p}}(x) \cup \operatorname{Crit}_{\mathrm{ep}}(x), \operatorname{Crit}_{\mathrm{ep}}(x) \neq \emptyset\right\}, \\
& J_{3}=\left\{x \in J(f): \operatorname{Crit}(x)=\operatorname{Crit}_{\mathrm{p}}(x) \neq \emptyset\right\} .
\end{aligned}
$$

Then $J(f)=\bigcup_{i=1}^{3} J_{i}$ and this is a classification of the points in $J(f)$.

Lemma $3.1(20)$. For any $x \in J_{1} \cup J_{2}$, there exist a puzzle piece $P_{0}$ of depth 0 and infinitely many $i_{n}$, such that

$$
\operatorname{deg}\left(f^{i_{n}}: P_{i_{n}}(x) \rightarrow P_{0}\right) \leqslant D
$$

for a constant $D<\infty$ independent of $i_{n}$.

Remark 3.2. Note that we can find a neighborhood $\widetilde{P}_{0}$ of $P_{0}$ satisfying $\overline{P_{0}} \subset \widetilde{P}_{0}$ and the annulus $\widetilde{P}_{0}-\overline{P_{0}}$ is disjoint from the critical orbits, since $J(f)$ is contained in the union of puzzle piece of depth 0 and the critical orbits in $F(f)$ are all attracted to the fixed point $\infty$.

The well-known Koebe Distortion Theorem plays an important role in the following proof. 
Theorem 3.3 (Koebe Distortion Theorem). Let $U, V$ be two simply connected domains in $\widehat{\mathbb{C}}$ satisfying $\bar{U} \subset V$ and let $g$ be a conformal map in $V$. Suppose the conformal modulus of $V-\bar{U}$ equals $\nu>0$. Then there is a constant $C \geqslant 1$ depending only on $\nu$ such that the inequalities

$$
\frac{1}{C} \leqslant \frac{\left|g^{\prime}(x)\right|}{\left|g^{\prime}(y)\right|} \leqslant C
$$

hold for all $x, y \in U$, where the derivative $g^{\prime}$ is considered in the spherical metric.

Definition 3.4. The density associated to $\mu$ of a set $X$ inside a set $Y$ is defined as

$$
\operatorname{dens}(X \mid Y)=\frac{\mu(X \cap Y)}{\mu(Y)} \text {. }
$$

We can conclude the following proposition immediately due to the definition of a conformal measure. See Proposition 3.5 in 12 .

Proposition 3.1. Let $U, V$ be the domains as in Theorem 3.3 and $\mu$ be a conformal measure for $f$ with exponent $\delta$. Suppose that $f$ is conformal in $V$. If $\mu$-measurable sets $A \subset B$ satisfy $A \subset U$ and $B \subset V$, then we have

$$
\frac{1}{K^{\delta}} \cdot \operatorname{dens}(A \mid B) \leqslant \operatorname{dens}(f(A) \mid f(B)) \leqslant K^{\delta} \cdot \operatorname{dens}(A \mid B),
$$

where $K$ is the constant in Theorem 3.3 .

Let

$$
\begin{aligned}
& X_{1}=\{x \in J(f): x \rightarrow c \text { for some } c \in \text { Crit }\} \cup \text { Crit, } \\
& X_{2}=J(f)-X_{1} .
\end{aligned}
$$

Obviously, $X_{2}$ consists of the non-critical points excluding critical points according to Definition 2.1(2).

Proposition 3.2. $\mu\left(X_{2}\right)=0$.

The following lemma is indispensable. Its proof is given by Prado; see Lemma 3.3 in 12 .

Lemma 3.5. For any set $X \subset J(f)$, if $\mu(X)>0$, then there is a point $x \in X$ such that

$$
\limsup _{n \rightarrow \infty} \operatorname{dens}\left(X \mid P_{n}(x)\right)=1 .
$$

Remark 3.6. Note that Lemma 3.5 is always true if we replace $\left\{P_{n}(x)\right\}$ by a subsequence $\left\{P_{n_{i}}(x)\right\}$.

Proof of Proposition 3.2. According to the classification of the points in $J(f)$, for each $x \in X_{2}$, there exists a smallest integer $k \geqslant 0$ such that $f^{n}(x) \notin \bigcup_{c \in \text { Crit }} P_{k}(c)$ for all $n>0$. Define

$$
X_{2, k}=\left\{x \in X_{2}: f^{n}(x) \notin \bigcup_{c \in \mathrm{Crit}} P_{k}(c), n>0\right\} .
$$

Then $X_{2}=\bigcup_{k=0}^{\infty} X_{2, k}$. We will prove that $\mu\left(X_{2, k}\right)=0$ for each $k \geqslant 0$ by contradiction. Assuming that $\mu\left(X_{2}\right)>0$, there must be some $k_{0}$ such that $\mu\left(X_{2, k_{0}}\right)>0$. By Lemma 3.5 there is a point $x \in X_{2, k_{0}}$ and a sequence of puzzle pieces $P_{n_{i}}(x)$, $n_{i} \rightarrow \infty$, such that $\operatorname{dens}\left(X_{2, k_{0}} \mid P_{n_{i}}(x)\right) \rightarrow 1$ as $n_{i} \rightarrow \infty$. 
Note that $f^{n_{i}-k_{0}}\left(P_{n_{i}}(x)\right)$ is a puzzle piece of depth $k_{0}$ and none of the puzzle pieces $\left\{P_{n_{i}}(x), f\left(P_{n_{i}}(x)\right), \ldots, f^{n_{i}-k_{0}}\left(P_{n_{i}}(x)\right)\right\}$ contain a critical point. Passing to a subsequence, we may assume $f^{n_{i}-k_{0}}\left(P_{n_{i}}(x)\right)=P_{k_{0}}\left(f^{n_{i}-k_{0}}(x)\right)=P_{k_{0}}$ for a fixed puzzle piece $P_{k_{0}}$ of depth $k_{0}$. Obviously,

$$
f^{n_{i}-k_{0}}: P_{n_{i}}(x) \rightarrow P_{k_{0}}
$$

is conformal for each $n_{i}$. Because $J(f)$ is contained in the union of puzzle pieces of depth $k_{0}$ and all the critical points outside $J(f)$ are attracted to $\infty$, we can choose a neighborhood $\widetilde{P}_{k_{0}}$ of $P_{k_{0}}$ such that $P_{k_{0}}$ is compactly contained in $\widetilde{P}_{k_{0}}$ and the annulus $\widetilde{P}_{k_{0}}-\overline{P_{k_{0}}}$ is disjoint from the critical orbits. So we can pull back $\widetilde{P}_{k_{0}}$ by $f^{n_{i}-k_{0}}$ along the orbit $x, f(x), \ldots, f^{n_{i}-k_{0}}(x)$ conformally. This implies that $f^{n_{i}-k_{0}}: P_{n_{i}}(x) \rightarrow P_{k_{0}}$ have bounded distortion with a constant independent of $n_{i}$. Making use of Proposition 3.1 and the fact that $\operatorname{dens}\left(X_{2, k_{0}} \mid P_{n_{i}}(x)\right) \rightarrow 1$, we conclude that $\operatorname{dens}\left(X_{2, k_{0}}^{c} \mid P_{k_{0}}\right)=0$, namely, $\operatorname{dens}\left(X_{2, k_{0}} \mid P_{k_{0}}\right)=1$. On the other hand, there exists some pre-image of critical puzzle pieces of depth 0 inside $P_{k_{0}}$, since the backward orbit of each point $x \in J(f)$ is dense in $J(f)$, so $\operatorname{dens}\left(X_{2, k_{0}} \mid P_{k_{0}}\right)$ should be bounded away from 1 . It is a contradiction. $\mu\left(X_{2}\right)=0$ is proved.

The proof of Proposition 3.2 follows the one given by Prado in [12].

\section{Proof of Main Theorem}

Above all, we will construct a sequence of nested Jordan domains containing $x$ for each $x \in X_{1}$ in which we can use the Koebe Distortion Theorem.

The following theorem is the main result in [2]. Due to this theorem, we are allowed to consider only the recurrent critical points.

Theorem 4.1. Suppose that $f$ is a rational map and $\mu$ is a conformal measure for $f$ with exponent $\delta$. Then exactly one of the following holds.

(1) The set of eventual pre-images of critical and parabolic periodic points in $J(f)$ together with the set of points $z \in J(f)$ such that $\omega(z)$ is a subset of the union of limit sets of recurrent critical points has full $\mu$-measure in $J(f)$.

(2) $\mu$ is ergodic, $\operatorname{supp}(\mu)=J(f), \omega(z)=J(f)$ for $\mu$-almost every point $z$ and $\delta$ is equal to the minimum exponent.

\section{Construction for critical points.}

(1) If $c \in \mathrm{Crit}_{\mathrm{p}}$, then for each $n \geqslant 0$, let $V_{n}(c)=K_{n}(c)$ and $\widetilde{V}_{n}(c)=\widetilde{K}_{n}(c)$, where $K_{n}(c)$ is the KSS nest. By Lemma 2.4, we know that $\bmod \left(\widetilde{V}_{n}(c)-\right.$ $\left.\overline{V_{n}(c)}\right)$ are uniformly bounded away from 0 . Moreover, all the annuli $\widetilde{V}_{n}(c)-$ $\overline{V_{n}(c)}$ are disjoint from the critical orbits.

(2) If $c \in$ Crit $_{\mathrm{r}}$, let $V_{n}(c)=P_{i_{n}}(c)$, where $P_{i_{n}}(c)$ is from Lemma 3.1 Recalling Remark 3.2, we know that for each $n \geqslant 0$, there is a neighborhood $\widetilde{P}_{i_{n}}(c)$ $\frac{\text { of } P_{i_{n}}}{P_{n_{n}}}(c)$ which is the pullback of $\widetilde{P}_{0}$ by $f^{i_{n}}$, such that the annulus $\widetilde{P}_{i_{n}}(c)-$ $\overline{P_{i_{n}}(c)}$ is disjoint from the critical orbits and the modulus of $\widetilde{P}_{i_{n}}(c)-\bar{P}_{i_{n}}(c)$ is bounded away from 0 . In this case, let $\widetilde{V}_{n}(c)=\widetilde{P}_{i_{n}}(c)$.

Briefly speaking, for each recurrent critical point $c$, there is a sequence of nested topological disks $V_{n}(c)$ such that for each $n \geqslant 0$ there exists a topological disk 
$\widetilde{V}_{n}(c) \supset \overline{V_{n}(c)}$ satisfying that $\widetilde{V}_{n}(c)-\overline{V_{n}(c)}$ is disjoint from the critical orbits and

$$
\bmod \left(\widetilde{V}_{n}(c)-\overline{V_{n}(c)}\right) \geqslant m,
$$

where $m>0$ is a constant independent of $n$ and $c$.

Construction for the points combinatorially convergent to recurrent critical points. For any $x \in J(f)$ which is combinatorially convergent to a recurrent critical point, let $l_{n}>0$ be the smallest integer such that $f^{l_{n}}(x) \in \bigcup_{c \in \mathrm{Crit}_{0}} V_{n}(c)$. Assume that $f^{l_{n}}(x) \in \widetilde{V}_{n}\left(c_{0}\right)$ for some $c_{0} \in \mathrm{Crit}_{0}$, denote by $W_{n}(x)$ the connected component of $f^{-l_{n}}\left(V_{n}\left(c_{0}\right)\right)$ containing $x$ and by $\widetilde{W}_{n}(x)$ the connected component of $f^{-l_{n}}\left(\widetilde{V}_{n}\left(c_{0}\right)\right)$ containing $x$. Due to the construction of $V_{n}(c)$ and $\widetilde{V}_{n}(c)$, it is not difficult to see that for each $n \geqslant 0, f^{l_{n}}: \widetilde{W}_{n}(x) \rightarrow \widetilde{V}_{n}\left(c_{0}\right)$ is conformal and $f^{l_{n}}$ has bounded distortion in $W_{n}(x)$.

Proof of Main Theorem. According to Theorem 4.1, if the set

$$
A=\left\{x \in X_{1}: x \rightarrow c \text { for some non-recurrent critical point } c\right\}
$$

has positive $\mu$-measure, then $\mu$ is ergodic and its exponent is the minimum exponent. Therefore, in the following proof, we only consider the points which are merely combinatorially convergent to recurrent critical points for convenience.

For each $n \geqslant 0$ and each critical point $c \in \mathrm{Crit}_{0}$, let $l_{n}$ be the landing time to $\bigcup_{c \in \mathrm{Crit}_{0}} V_{n}(c)$ of $x$ defined above. Define

$$
F_{n}(c)=\left\{x \in X_{1}: f^{l_{n}}(x) \in V_{n}(c), c \in \mathrm{Crit}_{0}\right\} .
$$

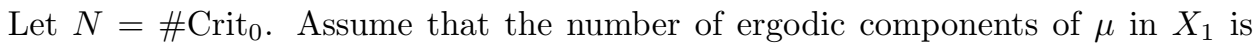
more than $N$. We pick $N+1$ ergodic components among them, called $E_{1}, \ldots$, $E_{N+1}$, so we get

$$
\bigcup_{c \in \text { Crit }_{0}} F_{n}(c)=X_{1} \text { and } 1=\sum_{c \in \text { Crit }_{0}} \mu\left(F_{n}(c)\right) \geqslant \sum_{i=1}^{N+1} \mu\left(E_{i}\right)
$$

for each $n \geqslant 0$. Let

$$
m_{0}=\min _{1 \leqslant i \leqslant N+1} \mu\left(E_{i}\right) .
$$

Then for arbitrary $n \geqslant 0$, there exist a critical point $c$ and subscripts $i, j \in$ $\{1, \ldots, N+1\}$ such that

$$
\mu\left(F_{n}(c) \cap E_{i}\right) \geqslant \frac{m_{0}}{N} \text { and } \mu\left(F_{n}(c) \cap E_{j}\right) \geqslant \frac{m_{0}}{N} .
$$

Otherwise, there exists an $i_{1}=i_{1}(c)$ such that

$$
\mu\left(F_{n}(c) \cap E_{i}\right)<\frac{m_{0}}{N}
$$

when $i \neq i_{1}$. We can conclude that there is a subscript $i_{0} \in\{1, \ldots, N+1\}$ satisfying

$$
\mu\left(F_{n}(c) \cap E_{i_{0}}\right)<\frac{m_{0}}{N}
$$

for all $c \in \mathrm{Crit}_{0}$. It is a contradiction that

$$
m_{0}=N \cdot \frac{m_{0}}{N}>\sum_{c \in \text { Crit }_{0}} \mu\left(F_{n}(c) \cap E_{i_{0}}\right)=\mu\left(E_{i_{0}}\right) \geqslant m_{0} .
$$

By passing to a subsequence, we can suppose that the above conclusion holds for a fixed critical point $c_{0}$ and fixed subscripts $i, j$ for each $n \geqslant 0$. 
Denote $F_{n}(c) \cap E_{i}$ by $I_{n}$. Note that

$$
\left\{J_{m}=\bigcup_{k=m}^{\infty} I_{k}\right\}_{m=0}^{\infty}
$$

is a decreasing sequence of subsets of $E_{i}$ and $\frac{m_{0}}{N} \leqslant \mu\left(J_{m}\right) \leqslant \mu\left(E_{i}\right)$ for all $m \geqslant 0$. Then we have

$$
\lim _{m \rightarrow \infty} J_{m}=\bigcap_{m=0}^{\infty} J_{m}=\bigcap_{m=0}^{\infty} \bigcup_{k=m}^{\infty} I_{k} \text { and } \mu\left(\bigcap_{m=0}^{\infty} \bigcup_{k=m}^{\infty} I_{k}\right) \geqslant \frac{m_{0}}{N} .
$$

Moreover, $\bigcap_{m=0}^{\infty} \bigcup_{k=m}^{\infty} I_{k}$ is the upper limit of the sets $\left\{I_{n}\right\}$ which consists of the points belonging to infinitely many $I_{n}$. So we can find a point $x \in F_{n_{k}}\left(c_{0}\right) \cap E_{i}$ such that

$$
\limsup _{k \rightarrow \infty} \operatorname{dens}\left(\left(F_{n_{k}}\left(c_{0}\right) \cap E_{i}\right) \mid W_{n_{k}}(x)\right)=1,
$$

where $n_{k}$ is a subsequence of $n$. In other words, we can find a subsequence $n_{k_{t}}$ of $n_{k}$ satisfying

$$
\lim _{t \rightarrow \infty} \operatorname{dens}\left(\left(F_{n_{k_{t}}}\left(c_{0}\right) \cap E_{i}\right) \mid W_{n_{k_{t}}}(x)\right)=1 .
$$

Now we consider $F_{n_{k_{t}}}\left(c_{0}\right) \cap E_{j}$. As above, we can find a subsequence of $n_{k_{t}}$, say $t_{m}$, and a point $y \in F_{t_{m}}\left(c_{0}\right) \cap E_{j}$ such that

$$
\lim _{m \rightarrow \infty} \operatorname{dens}\left(\left(F_{t_{m}}\left(c_{0}\right) \cap E_{j}\right) \mid W_{t_{m}}(x)\right)=1 .
$$

Note that $f^{l_{t_{m}}}: \widetilde{W}_{t_{m}}(x) \rightarrow \widetilde{V}_{t_{m}}\left(c_{0}\right)$ is conformal for every $m \geqslant 0$. Then by the Koebe Distortion Theorem we obtain that

$$
\frac{1}{K} \leqslant \frac{\left|\left(f^{l_{t_{m}}}\right)^{\prime}\left(z_{1}\right)\right|}{\left|\left(f^{l_{t_{m}}}\right)^{\prime}\left(z_{2}\right)\right|} \leqslant K
$$

for all $z_{1}$ and $z_{2}$ in $W_{t_{m}}(x)$, where $K$ is a constant independent of $m$.

According to Proposition 3.1 we have

$$
\frac{1}{K^{\delta}} \cdot \operatorname{dens}\left(E_{i}^{c} \mid W_{t_{m}}(x)\right) \leqslant \operatorname{dens}\left(E_{i}^{c} \mid V_{t_{m}}\left(c_{0}\right)\right) \leqslant K^{\delta} \cdot \operatorname{dens}\left(E_{i}^{c} \mid W_{t_{m}}(x)\right),
$$

since $E_{i}$ is $f$-invariant and $f^{l_{t_{m}}}\left(W_{t_{m}}(x)\right)=V_{t_{m}}\left(c_{0}\right)$, where $E_{i}^{c}$ is the complement of $E_{i}$. Hence, $\lim _{m \rightarrow \infty} \operatorname{dens}\left(E_{i}^{c} \mid W_{t_{m}}(x)\right)=0$ follows from the fact $\lim _{m \rightarrow \infty} \operatorname{dens}\left(E_{i} \mid W_{t_{m}}(x)\right)$ $=1$. Consequently, by the above inequalities, we have $\lim _{m \rightarrow \infty} \operatorname{dens}\left(E_{i}^{c} \mid V_{t_{m}}\left(c_{0}\right)\right)=0$ and $\lim _{m \rightarrow \infty} \operatorname{dens}\left(E_{i} \mid V_{t_{m}}\left(c_{0}\right)\right)=1$. Similarly, we also have $\lim _{m \rightarrow \infty} \operatorname{dens}\left(E_{j} \mid V_{t_{m}}\left(c_{0}\right)\right)=1$. This is a contradiction, because $E_{i} \cap E_{j}=\emptyset$ and $\operatorname{dens}\left(Y \mid V_{n}\left(c_{0}\right)\right)+\operatorname{dens}\left(Y^{c} \mid V_{n}\left(c_{0}\right)\right)=$ 1 for any set $Y \subset J(f)$ and each $n \geqslant 0$. Thus, $X_{1}$ contains at most $N$ ergodic components of $\mu$.

Remark 4.2. Actually, according to Theorem 4.1, we have the stronger result than the Main Theorem that the number of ergodic components of any conformal measure for $f \in \mathcal{F}$ is bounded by the number of recurrent critical points in the Julia set if there exists such a critical point or the conformal measure is ergodic.

\section{ACKNOWLEDGEMENTS}

The author would like to express his thanks to Prof. Yongcheng Yin for his suggestions and encouragement. He would also like to thank the referee for carefully reading the paper and providing valuable suggestions. 


\section{REFERENCES}

1. J. Aaronson, M. Denker and M. Urbański, Ergodic theory for Markov fibred systems and parabolic rational maps, Trans. Amer. Math. Soc., 337 (1993), 495-548. MR1107025 (94g:58116)

2. A. M. Blokh, J. C. Mayer amd L. G. Oversteegen, Recurrent critical points and typical limit sets for conformal measures, Topology Appl., 108 (2000), 233-244. MR.1794556 (2003d:37062)

3. M. Denker, R. D. Mauldin, Z. Nitecki and M. Urbański, Conformal measure for rational functions revisited, Fund. Math., 157 (1998), 161-173. MR.1636885 (99j:58122)

4. M. Denker and M. Urbański, On the existence of conformal measures, Trans. Amer. Math. Soc., 328 (1991), 563-587. MR1014246 (92k:58155)

5. M. Denker and M. Urbański, Absolutely continuous invariant measures for expansive rational maps with rationally indifferent periodic points, Forum Math., 3 (1991), 561-579. MR1129999 (92k:58154)

6. J. Graczyk and S. Smirnov, Non-uniform hyperbolicity in complex dynamics, Invent. Math., 175 (2009), 335-415. MR2470110 (2010e:37056)

7. O. Kozlovski, W. Shen and S. van Strien, Rigidity for real polynomials, Ann. of Math. (2), 165 (2007), 749-841. MR2335796 (2008m:37063)

8. J. Milnor, Dynamics in one complex variable: Introductory lectures, Third edition. Annals of Mathematics Studies, 160. Princeton University Press, 2006. MR2193309 (2006g:37070)

9. V. Mayer and M. Urbański, Geometric thermodynamical formalism and real analyticity for meromorphic functions of finite order, Ergod. Th. and Dynam. Sys., 28 (2008), 915-946. MR2422021 (2010f:37080)

10. V. Mayer and M. Urbański, Ergodic properties of semi-hyperbolic functions with polynomial Schwarzian derivative, Proceedings of the Edin. Math. Soc. (Series 2), 53 (2010), 471-502. MR.2653244

11. F. Przytycki, Iterations of holomorphic Collet-Eckmann maps: Conformal and invariant measures. Appendix: On non-renormalizable quadratic polynomials, Trans. Amer. Math. Soc., 350 (1998), 717-742. MR1407501 (98d:58155)

12. E. A. Prado, Ergodicity of conformal measures for unimodal polynomials, Conformal Geometry and Dynamics, 2 (1998), 29-44. MR1613051 (99g:58106)

13. F. Przytycki and J. Rivera-Letelier, Statistical properties of topological Collet-Eckmann maps, Ann. Sci. École Norm. Sup., 40 (2007), 135-178. MR2332354 (2008j:37093)

14. F. Przytycki and M. Urbański, Rigidity of tame rational functions, Bull. of the Polish Academy of Science: Mathematics, 47 (1999), 163-182. MR1686679 (2000i:37065)

15. W. Qiu and Y. Yin, Proof of the Branner-Hubbard conjecture on Cantor Julia sets, Sci. China Ser.A-Math., 52 (2009), 45-65. MR2471515 (2009j:37074)

16. D. Sullivan, Conformal dynamics systems. In: Geometric Dynamics. Springer Lecture Notes in Mathematics, 1007, Springer-Verlag, 1983, 725-752. MR730296 (85m:58112)

17. M. Urbański, Geometry and ergodic theory of conformal non-recurrent dynamics, Ergod. Th. and Dynam. Sys., 17 (1997), 1449-1476. MR.1488329 (99j:58178)

18. M. Urbański, Measures and dimensions in conformal dynamics, Bull. Amer. Math. Soc., 40 (2003), 281-321. MR1978566 (2004f:37063)

19. M. Urbański and A. Zdunik, Geometry and ergodic theory of non-hyperbolic exponential maps, Trans. Amer. Math. Soc., 359 (2007), 3973-3997. MR2302520 (2008j:37098)

20. Y. Yin and Y. Zhai, No invariant line fields on Cantor Julia sets, Forum Math., 22 (2010), 75-94. MR 2604364 (2011d:37077)

Department of Mathematics, School of Science, China University of Mining and Technology (Beijing), Beijing 100083, People's Republic of China

E-mail address: zhaiyu@amss.ac.cn 